\title{
Localized waves and mixed interaction solutions with dynamical analysis to the Gross-Pitaevskii equation in the Bose-Einstein condensate
}

Haotian Wang ( $\square$ haotian7624@126.com )

Beijing University of Posts and Telecommunications

Qin Zhou

Hubei Polytechnic University

Anjan Biswas

Alabama Agricultural and Mechanical University: Alabama A\&M University

Wenjun Liu

Beijing University of Posts and Telecommunications

\section{Research Article}

Keywords: Gross-Pitaevskii equation, Darboux transformation, Rogue wave, Breather, Mixed interaction solution, Numerical simulation

Posted Date: August 9th, 2021

DOl: https://doi.org/10.21203/rs.3.rs-786655/v1

License: (9) This work is licensed under a Creative Commons Attribution 4.0 International License. Read Full License 


\title{
Localized waves and mixed interaction solutions with dynamical analysis to the Gross-Pitaevskii equation in the Bose-Einstein condensate
}

\author{
Haotian Wang • Qin Zhou • Anjan Biswas • \\ Wenjun Liu
}

Received: date / Accepted: date

\begin{abstract}
We report a kind of breather, rogue wave and mixed interaction structures on a variational background height in the Gross-Pitaevskii equation in the Bose-Einstein condensate by the generalized Darboux transformation method, and the effects of related parameters on rogue wave structures are discussed. Numerical simulation can discuss the dynamics and stability of these solutions. We numerically confirm that these are correct, and can be reproduced from a deterministic initial profile. Results show that rogue waves and mixed interaction solutions can evolve with a small amplitude perturbation under the initial profile conditions, but breathers cannot. Therefore, these can be used to anticipate the feasibility of their experimental observation.
\end{abstract}

Keywords Gross-Pitaevskii equation · Darboux transformation · Rogue wave · Breather · Mixed interaction solution · Numerical simulation

\section{Introduction}

As a new state of matter and a field in physics, the surge of interest in Bose-Einstein condensate (BEC) has been prompted in recent years by researchers [1-7]. The mean-field Gross-Pitaevskii equation (GPE) is used to describe and understand some nonlinear phenomena, interesting and important properties and characteristics of vortex states [8-12] and localized waves including soliton [13-16], rogue wave (RW) [1719], breather [20,21] in BEC, which is also one of the main theoretical research methods, and some predictions have been exposed to agree with relevant vortex and localized wave experiments [22-24]. The GPE is similar to the famous nonlinear Schrödinger equation (NLSE), the former is a special variant of the latter, and the latter is widely related to applications in many fields such as optics, quantum mechanics,

H. Wang · W. Liu (凶)

State Key Laboratory of Information Photonics and Optical Communications, and School of Science, Beijing University of Posts and Telecommunications, P. O. Box 122, Beijing 100876, People's Republic of China

E-mail: jungliu@bupt.edu.cn

Q. Zhou

School of Mathematics and Physics, Hubei Polytechnic University, Huangshi 435003, People's Republic of China

Q. Zhou

Nonlinear Analysis and Applied Mathematics (NAAM)-Research Group, Department of Mathematics, Faculty of Science, King Abdulaziz University, P.O. Box 80257, Jeddah 21589, Saudi Arabia

A. Biswas

Department of Physics, Chemistry and Mathematics, Alabama A\&M University, Normal, AL 35762-4900, USA

A. Biswas

Mathematical Modeling and Applied Computation (MMAC) Research Group, Department of Mathematics, King Abdulaziz University, Jeddah-21589, Saudi Arabia

A. Biswas

Department of Applied Mathematics, National Research Nuclear University, 31 Kashirskoe Hwy, Moscow-115409, Russian Federation

A. Biswas

Department of Mathematics and Applied Mathematics, Sefako Makgatho Health Sciences University, Medunsa-0204, Pretoria, South Africa 
quantum field theory, biophysics, fluid dynamics, plasma physics and so on. [25]. It is interesting that, from the mathematical viewpoint of $\mathrm{BEC}$, interatomic interaction may be used to derive the nonlinearity of the GPE which can be explained by the efficient mean-field [26-30]. All that matters is that the study on GPEs allows to predict and describe some important, interesting, and experimentally relevant, nonlinear effects, localized waves such as solitons, breathers, RWs, and nonlinear states such as domainwalls, vortices. The GPE can accurately describe the macroscopic wave function dynamics in the ground state of BEC of interacting particles, and a lot of other phenomena and effects such as the interference properties, condensate expansion, collective excitations, sound propagation, etc [31].

Localized waves are very important nonlinear phenomena, and include, mainly, solitons, RWs and breathers. So far, localized waves have been found in many fields such as nonlinear optics, biophysics, plasmas, oceanophysics, hydromechanics, and BECs [32-38]. Therefore, creating nonlinear localized waves in these fields has motivated intense attention and interest in their properties and abundant structures [3943]. In recent years, localized waves in BEC have also attracted the attention of researchers [13-21]. The exact localized wave solutions and time evolution of them in BECs are described by the GPE with abundant different external potentials. In a general system, the localized waves are spatially, temporally (solitons and breathers) or spatiotemporally (RWs) localized, and will arise from vanishing or nonvanishing backgrounds, however, these solutions may also exhibit non-localized phenomena, or appear in a variable background height in some special systems, e.g. nonautonomous systems [44,45]. Considering the Gross-Pitaevskii equation (GPE) as [7]

$$
\mathrm{i} \hbar \frac{\partial}{\partial t} \psi(\mathbf{r}, t)=\left[-\frac{\hbar^{2}}{2 m} \nabla^{2}+g|\psi(\mathbf{r}, t)|^{2}+V_{\mathrm{ext}}(\mathbf{r})\right] \psi(\mathbf{r}, t)
$$

where $\hbar$ is the Planck constant, $\psi=\psi(\mathbf{r}, t)$ is the macroscopic wave function, $m$ is the mass of an atom, and external potential $V_{\text {ext }}=V_{\text {ext }}(\mathbf{r}), \mathbf{r}$ is $n$-dimensional vector, $\mathrm{i}^{2}=-1$. The coefficient $g$ of nonlinearity strength in the GP is given by $g=\frac{4 \pi \hbar a}{m}$, which may be negative or positive, and this is because the scattering length is different, in the other word, one can be $a<0$ (for Li BEC) or $a>0$ (for $\mathrm{Rb}$ or $\mathrm{Na}$ BEC) [7]. Set $\mathbf{r}=x, g=-2 u^{2} \hbar, m=\frac{\hbar}{2}, V_{\text {ext }}(\mathbf{r})=-\hbar\left(\mathrm{i} \beta-\alpha x+\beta^{2} x^{2}\right)$, GPE (1) can be transformed into an integrable one-dimensional nonautonomous GPE that can describe the macroscopic ground state wave function dynamics in BEC as follows [44,46,47]

$$
\mathrm{i} \frac{\partial}{\partial t} \psi=-\left(\frac{\partial^{2}}{\partial x^{2}}+2 u^{2}|\psi|^{2}+\mathrm{i} \beta-\alpha x+\beta^{2} x^{2}\right) \psi,
$$

where the $\alpha$ and $\beta$ are real parameters in external potentials, and the coefficient $u$ is the nonlinearity strength in the GPE (2). The Eq. (2) can describe macroscopic waves in some atom BECs such as Li BEC due to the $a=-\frac{\hbar u^{2}}{4 \pi}<0$ for free real nonlinearity strength $u$. In fact, the $V_{\text {ext }}(\mathbf{r})$ can be divided into three parts including linear external potential $-\alpha x$, parabolic external potential $\beta^{2} x^{2}$, and constant term $\mathrm{i} \beta$. It needs to be pointed out that it can be gain and loss terms for $b<0$ and $b>0$, respectively. Hence, for Eq. (2), there are several cases in the BEC [44]: (i) When $\alpha=0$, Eq. (2) can be used to describe the dynamics of wave functions with gain (or loss) term and parabolic external potential [47,48]; (ii) When $\beta=0$, Eq. (2) can be used to describe the dynamics of wave functions with linear external potential, and without gain (or loss) term; (iii) When $\mu=1, \alpha \neq 0, \beta \neq 0$, Eq. (2) can describe the dynamics of wave functions with gain (or loss) term, linear external potential and parabolic external potential, constant interatomic interactions; (iv) When $\mu=1, \alpha=\beta=0$, Eq. (2) reduce to the NLSE which controls the dynamics of wave function without gain (or loss) term and external potential.

The GPE (2) has a Lax pair as [44]

$$
\Phi_{\mathrm{x}}=\frac{\partial}{\partial x} \Phi=L \Phi, \quad \Phi_{\mathrm{t}}=\frac{\partial}{\partial t} \Phi=V \Phi
$$

with

$$
\begin{aligned}
& L=-\mathrm{i} \sigma_{3} \lambda(t)+u U, \\
& V=-2 \mathrm{i} \sigma_{3} \lambda^{2}(t)+\left(2 \mathrm{i} \beta x \sigma_{3}+2 u U\right) \lambda(t)-\left(i u^{2} \sigma_{3} U^{2}+\frac{\mathrm{i}}{2} \alpha x \sigma_{3}+2 \beta u x U-\mathrm{i} u \sigma_{3} U_{x}\right),
\end{aligned}
$$

where $\Phi=(\varphi, \zeta)^{\mathrm{T}}, U=\left(\begin{array}{cc}0 & Q \\ -Q^{*} & 0\end{array}\right), \sigma_{3}=\left(\begin{array}{cc}1 & 0 \\ 0 & -1\end{array}\right)$ is the Pauli matrix, $U_{x}=\frac{\partial}{\partial x} U, Q=\psi \mathrm{e}^{-\frac{\mathrm{i} \beta x^{2}}{2}}$, $\lambda(t)=\xi \mathrm{e}^{-2 \beta t}+\frac{\alpha}{4 \beta}, \xi$ is the spectrum parameter, and the asterisk means the complex conjugate.

This paper includes four sections. The Darboux transformation (DT) $[49,50]$ and exact solutions of Eq. (2) are constructed in Section 2. Then, in Section 3, the structures of higher-order RW solutions, 
breather solutions, and localized wave mixed interaction solutions in some different backgrounds are plotted and their dynamical properties are discussed by numerical simulation. The conclusion in the last section will summarize the whole paper.

\section{Darboux transformation and exact solutions}

\subsection{Darboux transformation}

To obtain the exact solutions, first of all, a necessary $N$-fold DT matrix needs to be constructed. For Lax pair (3), we consider as following the gauge transformation

$$
\widetilde{\Phi}=T \Phi,
$$

based on the knowledge of DT, the Darboux matrix $T$ is described below

$$
T=\xi^{N} \mathrm{I}_{2}+\sum_{j=0}^{N-1} \Gamma^{(j)} \xi^{j}
$$

where $\Gamma^{(j)}=\left(\begin{array}{cc}f^{(j)} & g^{(j)} \\ -g^{(j)^{*}} & f^{(j)^{*}}\end{array}\right)$, the $N$ is a nonzero positive integer and $\mathrm{I}_{2}$ is the second-order unit matrix, $f^{(j)}$ and $g^{(j)}$ are functions that depend on $x$ and $t$ and can be solved by $T\left(\xi_{i}\right) \Phi_{i}\left(\xi_{i}\right)=0$. The DT matrix can make the $\widetilde{\Phi}$ satisfy $\widetilde{\Phi}_{x}=\widetilde{L} \widetilde{\Phi}, \widetilde{\Phi}_{t}=\widetilde{V} \widetilde{\Phi}$, while $\widetilde{L}=\left(T L+T_{x}\right) T^{-1}, \widetilde{V}=\left(T V+T_{t}\right) T^{-1}$ have the same forms as $L$ and $V$ in Lax pair (3), except replacing the original solution $q$ with new one $\widetilde{q}$, namely,

$$
\widetilde{q}=\frac{u q+2 \mathrm{i} g^{(N-1)} \mathrm{e}^{\frac{\beta\left(\mathrm{ix} x^{2}-4 t^{2}\right)}{2}}}{u}
$$

This is a well-known step for the most standard $N$-fold DT. However, the method cannot be used to get the rational solution such as RW, hence, we need to extend it to generalized DT (i.e. generalized $(m, N-m)$-fold DT $[49,50])$. Choosing some appropriate expansion methods of $\left.T\left(\xi_{i}\right) \Phi_{i}\left(\xi_{i}\right)\right|_{\xi_{i} \rightarrow \xi_{i}+z}=0$, namely, $T\left(\xi_{i}+z\right) \Phi\left(\xi_{i}+z\right)=\left[T^{(0)}+T^{(1)} z+\cdots+T^{\left(m_{i}\right)} z^{m_{i}}\right]\left[\Phi^{(0)}\left(\xi_{i}\right)+\Phi^{(1)}\left(\xi_{i}\right) z^{2}+\Phi^{(2)}\left(\xi_{i}\right) z^{4}+\cdots+\right.$ $\left.\Phi^{\left(m_{i}\right)}\left(\xi_{i}\right) z^{2 m_{i}}+O\left(z^{2 m_{i}+2}\right)\right]=0$, where $z$ is a small parameter, $T^{\left(m_{i}\right)}$ and $\xi^{\left(m_{i}\right)}$ are obtained via binomial expansion and Taylor expansion, respectively. Then, the $f^{(j)}$ and $g^{(j)}$ in the following systems can be solved through Cramer's Rule:

$$
\sum_{j=0}^{r_{i}} T^{(j)}\left(\xi_{i}\right) \Phi^{\left(r_{i}-j\right)}\left(\xi_{i}\right)=0, \quad i=1,2, \cdots, m, \quad r_{i}=0,1,2, \cdots, m_{i}
$$

which is a system of $2 N$ equations $\left(N=\sum_{i=1}^{m}\left(1+m_{i}\right)\right)$, the $m_{i}$ is the highest-order derivative of $\left.\xi\left(\xi_{i}\right)\right|_{\xi_{i}=\xi_{i}+z}$ in $\left.T\left(\xi_{i}\right) \xi\left(\xi_{i}\right)\right|_{\xi_{i}=\xi_{i}+z}=0$. In fact, the $m_{i}+1$ is the multiplicity of the root $\xi_{i}$ of equation $|T(\xi)|=0$. Readers can refer to Refs. $[49,50]$ for details of the generalized DT method.

\subsection{Exact solutions}

By the generalized $(m, N-m)$-fold DT, several kinds of analytical solutions including RW, breather, mixed interaction of localized waves can be produced in this part. Substituting the plane-wave seed solution $q=A_{c} \mathrm{e}^{\frac{h(x, t)}{4 \beta^{2}}}$ into $(3)$, where $h(x, t)=-2 \mathrm{i} \beta\left(A_{c}^{2} u^{2}-\frac{\omega_{c}^{2}}{2}\right) \mathrm{e}^{-4 \beta t}+4 \mathrm{i}\left(\beta^{2} x-\frac{\alpha}{2}\right) \omega_{c} \mathrm{e}^{-2 \beta t}+\left(2 \mathrm{i} x^{2}-\right.$ $8 t) \beta^{3}-2 \mathrm{i} \alpha \beta x-\mathrm{i} \alpha^{2} t$, we can give a group of solution as

$$
\Phi(\xi)=\left[\begin{array}{c}
\left(C_{i} \mathrm{e}^{A}+D_{i} \mathrm{e}^{-A}\right) \mathrm{e}^{F} \\
\left(C_{i} M_{+} \mathrm{e}^{A}+D_{i} M_{-} \mathrm{e}^{-A}\right) \mathrm{e}^{-F}
\end{array}\right],
$$


with

$$
\begin{aligned}
& a(t)=\omega_{c} \mathrm{e}^{-2 \beta t}-\frac{\alpha}{2 \beta}, \quad b(t)=-\frac{\mathrm{e}^{-4 \beta t}\left(2 \omega_{c} \alpha \mathrm{e}^{2 \beta t}+\alpha^{2} \mathrm{e}^{4 \beta t} t-\omega_{c}^{2} \beta+2 A_{c}^{2} \beta u^{2}\right)}{4 \beta^{2}}, \\
& A=\frac{\mathrm{i} \eta\left[4 \beta^{2} x-2 \alpha+\left(-2 \beta \xi+\omega_{c} \beta\right) \mathrm{e}^{-2 \beta t}\right] \mathrm{e}^{-2 \beta t}+\eta \Delta}{8 \beta^{2}}, \quad F=\frac{\mathrm{i}[b(t)+a(t) x]}{2}, \\
& M_{ \pm}=\frac{\mathrm{i}\left(\omega_{c} \pm \eta+2 \xi\right)}{2 A_{c} u}, \quad \eta=\sqrt{4 A_{c}^{2} u^{2}+4 \xi^{2}+4 \omega_{c} \xi+\omega_{c}^{2}}, \quad \Delta=\sum_{j=0}^{N-1}\left(\mu_{i}+\mathrm{i} \nu_{i}\right) z^{j},
\end{aligned}
$$

where $C_{i}, D_{i}$ are complex constants, $A_{c}, \omega_{c}, \mu_{i}$ and $\nu_{i}$ are free real numbers. It should be pointed out that the parameters $\mu_{i}$ and $\nu_{i}$ can make the higher-order RW separate into many lower-order RWs. Then, let's substitute the spectral number $\xi=M+z^{2}$ with $M=-\frac{\omega_{c}}{2}+\mathrm{i} A_{c} u$ and $C_{1}=-D_{1}=\frac{1}{z}$ into Eq. (9), we can give a group of expression of rational functions via Taylor expansion around $z=0$, namely,

$$
\Phi\left(z^{2}\right)=\sum_{k=0}^{\infty} \Phi^{(k)} z^{2 k}=\Phi^{(0)}+\Phi^{(1)} z^{2}+\Phi^{(2)} z^{4}+\Phi^{(3)} z^{6}+\cdots
$$

Then, form the (7), an exact solution of GPE (2) is given by

$$
q=\frac{u A_{c} \mathrm{e}^{\frac{h(x, t)}{4 \beta^{2}}}+2 \mathrm{i} g(N-1) \mathrm{e}^{\frac{\beta\left(\mathrm{i} x^{2}-4 t^{2}\right)}{2}}}{u},
$$

the $g^{(N-1)}$ can be solved through the following equation group

$$
\left\{\begin{array}{l}
\sum_{j=0}^{r_{l}} T^{(j)}\left(\xi_{l}\right) \Phi^{\left(r_{l}-j\right)}\left(\xi_{l}\right)=0, \quad l=1,2, \cdots, n, \quad 0 \leq n \leq m, \quad r_{l}=0,1,2, \cdots, m_{l}, \\
T\left(\xi_{n+k}\right) \Phi\left(\xi_{n+k}\right)=0, \quad k=1,2, \cdots, m-n .
\end{array}\right.
$$

Remark 1. We divide $m$ different spectral parameters into two parts: $\Xi_{1}=\left\{\xi_{1}, \xi_{2}, \cdots, \xi_{l}, \cdots, \xi_{n}\right\}$ and $\Xi_{2}=\left\{\xi_{n+1}, \xi_{n+2}, \cdots, \xi_{n+k}, \cdots, \xi_{m}\right\}$. For system (12), the spectral parameters in set $\Xi_{1}$ are represented as $\xi=\xi_{l}+z^{2}$ and substituted into the expression (9), respectively, and then expand them as (10) via Taylor series expansion. The $n$ represents the number of parameters that need to be substituted and expanded. In addition, the spectral parameters in set $\Xi_{2}$ are substituted into (9), respectively, without Taylor series expansion. The term $N-m$ in generalized $(m, N-m)$-fold DT equals to $\sum_{l=1}^{n} m_{l}$, in other words, it represents the sum of the highest derivatives in Taylor series expansion corresponding to each spectral parameter. When we choose $m$ appropriate spectral parameters, different $g^{(N-1)}$ form system (12) can be obtained and it may give abundant localized wave structures.

\section{Discussion}

In this section, we will discuss the localized wave structures with some special parameters:

(I) When $m=n=1$ (i.e. $\Xi_{1}=\left\{\xi_{1}\right\}$ and $\Xi_{2}=\varnothing$ ) and $m_{1}=0$, set $\alpha=0, \beta=-1, u=2, A_{c}=2$, $\omega_{c}=0, \xi_{1}=M, C_{1}=-D_{1}=\frac{1}{z}, \mu_{0}=12, \nu_{0}=0$, the wave function $|\psi|$ is a first-order RW on a growing plane-wave background height, and the structure can be plotted in Fig. 1 (a1). The special type of RW solutions on a growing plane-wave background height of the GPE (2) can be found to be

$$
\psi=-2 \mathrm{e}^{2 t-\frac{\mathrm{i} x^{2}}{2}+8 \mathrm{ie}^{4 t}}-2 \mathrm{e}^{2 t-\frac{\mathrm{i} x^{2}}{2}+8 \mathrm{ee}^{4 t}} \frac{-32 \mathrm{ie}^{4 t}-2+48 \mathrm{i}}{32 \mathrm{e}^{4 t} x^{2}-8 \mathrm{e}^{2 t} x+128 \mathrm{e}^{8 t}-384 \mathrm{e}^{4 t}+289} .
$$

Figure 1 (b1) shows the contour maps of RW (13) and the lines with different colors indicate the different amplitudes. It is obvious that the background value is increasing along the $t$-axis. Change the parameters $\alpha=1, u=1, A_{c}=1, \omega_{c}=-1, \mu_{0}=8$, the structure and corresponding contour map of RW solution (11) are shown in Fig. 1 (a2)(b2). These changed parameters make the RW have a new direction of propagation.

Figure 2 exhibits the evolutions of the peak of RW under some distinct parameters. Choose the parameters $\alpha=1, \beta=-1, A_{c}=2, \omega_{c}=0, \mu_{0}=15, \nu_{0}=0$, and Fig. 2 (a) shows peak of six different RWs with distinct nonlinearity strength $\mu$. It is easy to see that the nonlinear strength $\mu$ does not affect the height and growth rate of the amplitude of the background, but does affect the peak value and the 
(a1)

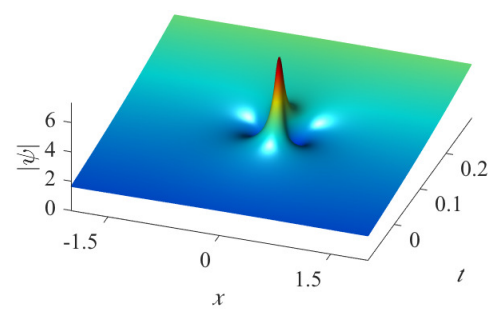

(b1)

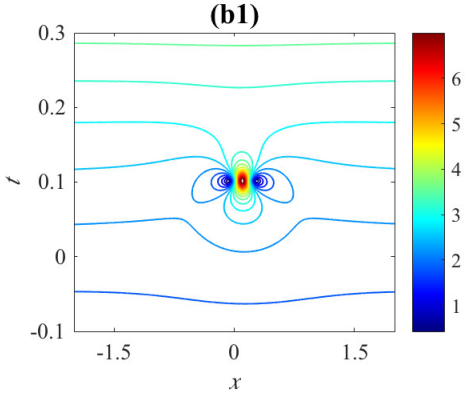

(a2)

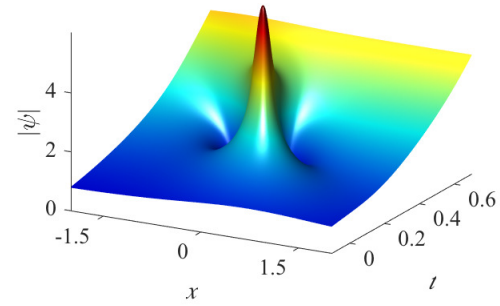

(b2)

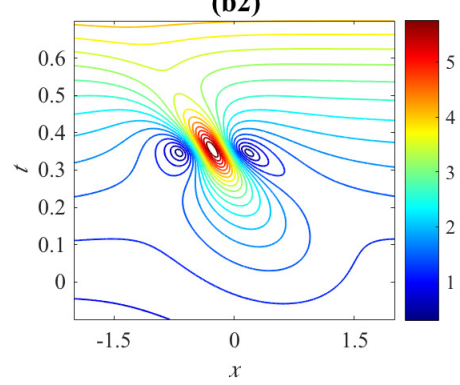

(a3)

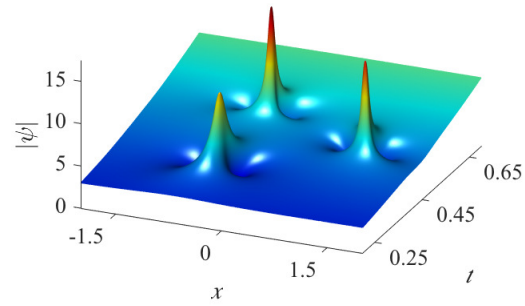

(b3)

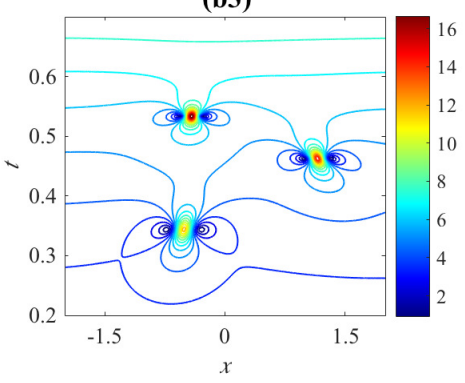

Fig. 1 (a1)-(a3) The RW structures and (b1)-(b3) their contour maps on a growing plane-wave background height with the same parameters $\beta=-1, \xi_{1}=-\frac{\omega_{c}}{2}+\mathrm{i} A_{c} u, C_{1}=-D_{1}=\frac{1}{z}$ and different parameters (a1)(b1) First-order RW: $\alpha=0, u=2, A_{c}=2, \omega_{c}=0, \mu_{0}=12, \nu_{0}=0$; (a2)(b2) First-order RW: $\alpha=1, u=1, A_{c}=1, \omega_{c}=-1, \mu_{0}=8, \nu_{0}=0$; (a3)(b3) Second-order RW: $\alpha=0, u=1, A_{c}=2, \omega_{c}=0, \mu_{0}=25, \mu_{1}=100, \nu_{0}=\nu_{1}=0$.

(a)

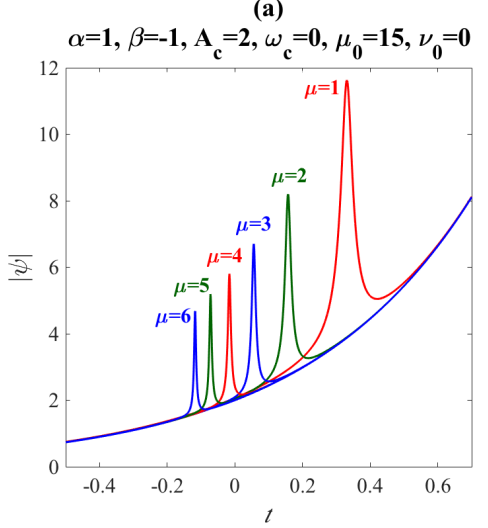

(b)

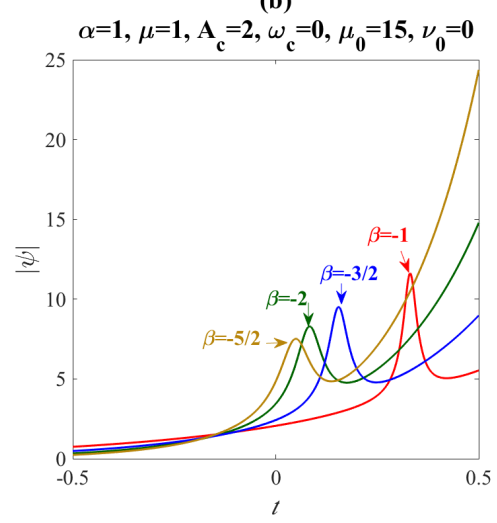

(c)

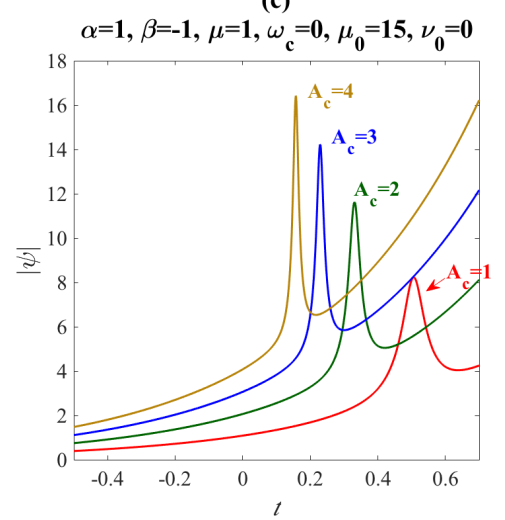

Fig. 2 (Color online) The evolution of peak of RW with distinct parameters.

initial phase of the RW. As the strength increases, the amplitude of RW decreases, and its occurrence moves along the negative $t$-axis. When $\mu<0$, no RW is found in Eq. (2). When we choose the parameters $\alpha=1, \mu=1, A_{c}=2, \omega_{c}=0, \mu_{0}=15, \nu_{0}=0$, and evolutions of the peak of four different RWs with different coefficient $\beta$ of parabolic external potential with a gain term $(\beta<0)$ is exhibited in Fig. 2 (b). The result shows that the larger the value of $|\beta|$, the faster the growth rate of background height, and the smaller the peak value of RW. In particular, no RW is found when the parabolic external potential has a loss term $(\beta>0)$. Fig. 2 (c) exhibits the evolutions of the peak of four different RWs with different background height $A_{c}$, result shows that the larger the value of $A_{c}$, the faster the growth rate of background height, and the larger the peak value of RW. In addition, the coefficient $\alpha$ of linear external potential and the background frequency $\omega_{c}$ do not have an evident effect on the structure of RW and the background height.

The RW shape wave function $|\psi|$ of such a kind has one local maximum point around $|\psi| \max =$ $(0.10201,0.10161)$ and two local minimums points $|\psi|_{\min , \pm}=\left(\frac{\sqrt{6}}{24} \pm \frac{\sqrt{2}}{8}, \frac{1}{4} \ln \frac{3}{2}\right)$, and their corresponding profile maps are exhibited in Fig. 4 (a1)(b1). The deep red line (for $x=\frac{1}{8 \mathrm{e}^{2 t}}$ ) and blue line (for $x=$ $\frac{\sqrt{6}}{24}+\frac{\sqrt{2}}{8}$ ) in Fig. 4 (a1) show the profiles of RW structure $|\psi|$. The red line in Fig. 4 (b1) shows the profile of RW structure $|\psi|$ when $t=\frac{1}{4} \ln \frac{3}{2}$ in solution (13). According to the above analysis, the RW solution 
has a localized peak value of around 7.35 at the point $|\psi|_{\max }$ and a localized depression value of 0 at two points $|\psi|_{\text {min }, \pm}$. For time $t=\frac{1}{4} \ln \frac{3}{2}$, the RW peak amplitude of the $\psi$ field becomes now around 3.5 times the background height (Fig. 4 (b1)), and there is a conservation law as $\int_{-\infty}^{\infty}\left(|\widetilde{\psi}|-\psi_{B g}\right) \mathrm{d} x=0$, the $\psi_{B g}$ represents the background height at different time $t$. Therefore, the number of atoms in the field can be conserved even if their macroscopic wave functions show abundant localized wave structures. As time goes on, RW will disappear from the field, however, the background height will continue to grow.

(a1)

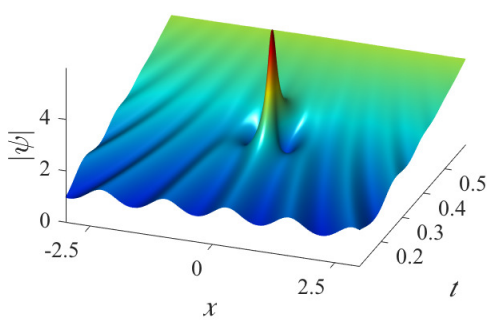

(b1)

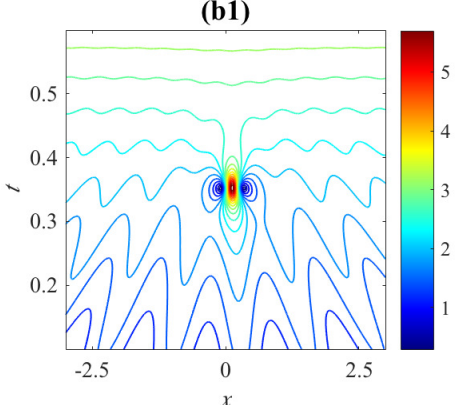

(a2)

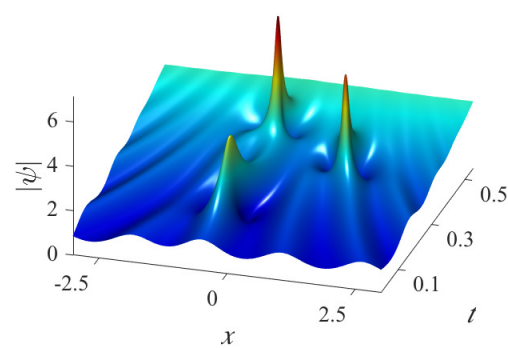

(b2)

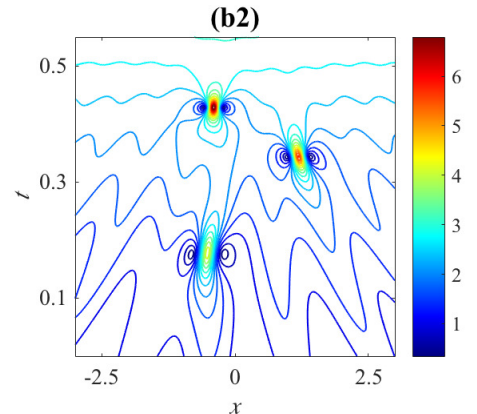

(a3)

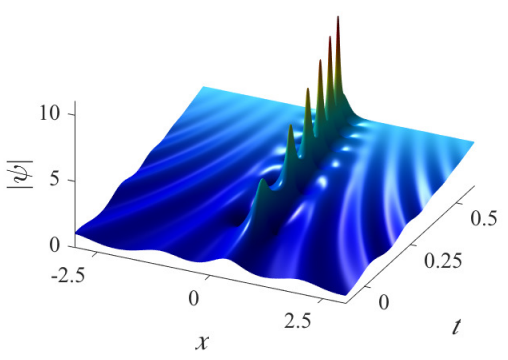

(b3)

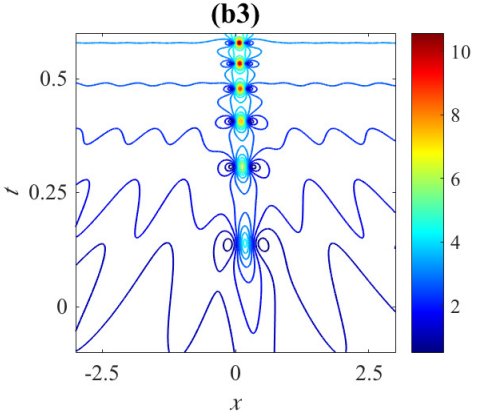

Fig. 3 (a1)(a2) The RW structures, (a3) nonautonomous breather structure and (b1)-(b3) their contour maps on a growing periodic-wave background with the same parameters $\alpha=0, \beta=-1, u=2, A_{c}=1, \omega_{c}=0, \xi_{2}=\frac{i}{3}$ and different parameters (a1)(b1) First-order RW: $C_{1}=-D_{1}=\frac{C_{2}}{z}=\frac{D_{2}}{z}=\frac{1}{z}, \xi_{1}=-\frac{\omega_{c}}{2}+\mathrm{i} A_{c} u, \mu_{0}=16, \nu_{0}=0$; (a2)(b2) Second-order RW: $C_{1}=-D_{1}=\frac{C_{2}}{z}=\frac{D_{2}}{z}=\frac{1}{z}, \xi_{1}=-\frac{\omega_{c}}{2}+\mathrm{i} A_{c} u, \mu_{0}=15, \mu_{1}=50, \nu_{0}=\nu_{1}=0$; (a3)(b3) Nonautonomous breather: $C_{1}=D_{1}=C_{2}=-D_{2}=1, \xi_{1}=\frac{5 \mathrm{i}}{2}$.

(II) When $m=n=1$ (i.e. $\Xi_{1}=\left\{\xi_{1}\right\}$ and $\Xi_{2}=\varnothing$ ) and $m_{1}=1$, set $\alpha=0, \beta=-1, u=1, A_{c}=2$, $\omega_{c}=0, \xi_{1}=M, C_{1}=-D_{1}=\frac{1}{z}, \mu_{0}=25, \mu_{1}=100, \nu_{0}=\nu_{1}=0$, the wave function $|\psi|$ is second-order RW (three first-order RWs) on a growing plane-wave background height and its corresponding structure and contour map are plotted in Fig. 1 (a3)(b3), and the expression of the wave function $|\psi|$ is listed in Appendix. Due to $m_{1}=1$, the structure will include three first-order RWs. Affected by the increase of background height over time, the amplitudes of the three RWs at different moments are different. Even so, each RW still exists a conservation law like (I), and when they reach the highest point, each RW peak amplitude at the different moment of the field is still about 3.5 times the background height at the current time. Hence, the generation of multiple RW in the field at corresponding moments can be described and simulated in BEC by macroscopic wave function $\psi$ of the GPE (2), the atoms in the field condense more times and the number of them maintains conservation. The first- and second-order RW solutions in Fig. 1 are spatiotemporally localized, apparently, which differ from the nonautonomous RW in Ref. [44].

(III) When $m=2, n=1$ (i.e. $\Xi_{1}=\left\{\xi_{1}\right\}$ and $\Xi_{2}=\left\{\xi_{2}\right\}$ ), $m_{1}=0$, set $\alpha=0, \beta=-1, u=2, A_{c}=1, \omega_{c}=0$, $\xi_{1}=M, \xi_{2}=\frac{\mathrm{i}}{3}, C_{1}=-D_{1}=\frac{1}{z}, C_{2}=D_{2}=1, \mu_{0}=16, \nu_{0}=0$, the first-order RW-shape wave function $|\psi|$ structure on a growing periodic-wave background height and its corresponding contour map can be exhibited in Fig. 3 (a1)(b1). Besides, the spectral parameter $\xi_{2}$ is controllable and can affect the structure of the periodic-wave which presents a nonautonomous periodic-wave [44]. This kind of RW structure is similar to Fig. 1 (a1), except for the background. In fact, it also may be understood as the interaction of periodic-wave and RW. The RW has one localized peak value about 5.9 near the point around $(0.13,0.35)$, and a localized minimum value 0 at two depression points. The deep red line (for $x \approx 0.13$ ) and the blue 
line (for $x \approx 0.34$ ) in Fig. 4 (a2) show the profiles of RW structure $|\psi|$. The red line in Fig. 4 (b2) shows the profile of RW structure $|\psi|$ when $t \approx 0.35$. It is clear that the two lines in Fig. 4(a1) will overlap initially and then evolve into trajectories of maximum and minimum values respectively, while the two lines in Fig. 4(a2) will not overlap initially (see yellow circles in Fig. 4). This also shows the difference in background shape. Change the parameters $m_{1}=1, \mu_{0}=15, \mu_{1}=50$ and $\nu_{0}=\nu_{1}=0$, the structure and corresponding contour map of second-order RW-shape wave function $|\psi|$ are exposed in Fig. 3 (a2)(b2). Energy of atoms in the field can be conserved at the peak of RW according to the macroscopic wave structure. As time goes on, RW will disappear from the field, however, the background height will continue to grow.

(IV) When $m=2, n=0$ (i.e. $\Xi_{1}=\varnothing$ and $\Xi_{2}=\left\{\xi_{1}, \xi_{2}\right\}$ ), set $\alpha=0, \beta=-1, u=2, A_{c}=1, \omega_{c}=0$, $\xi_{1}=\frac{5 \mathrm{i}}{2}, \xi_{2}=\frac{\mathrm{i}}{3}, C_{1}=D_{1}=C_{2}=-D_{2}=1$, the one-breather structure on a growing periodic-wave background and corresponding contour map can be exhibited in Fig. 3 (a3)(b3), and it exhibits the dynamics of the bright breather in an attractive parabolic potential. From Fig. 3 (a3)(b3), it can be seen that with the increase of time $t$ and background height, the peak value of the bright breather increases and the width is compressed. It can give a bright breather with the assumed peak value for wave function $|\psi|$.

(a1)

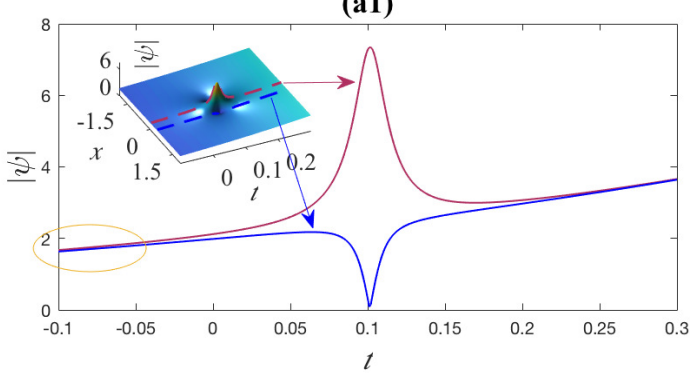

(a2)

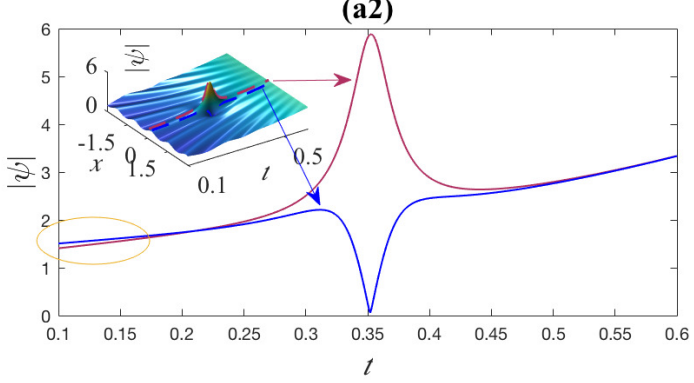

(b1)

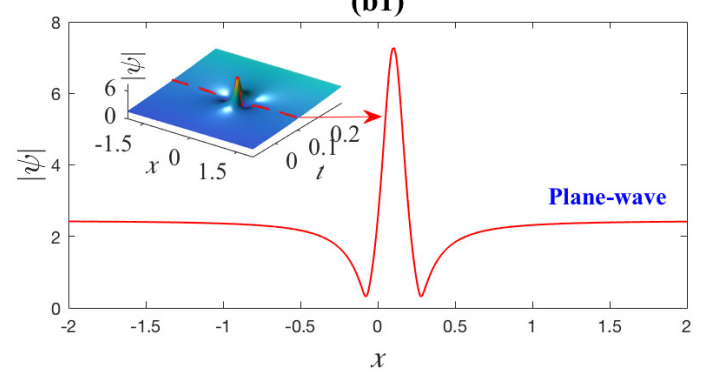

(b2)

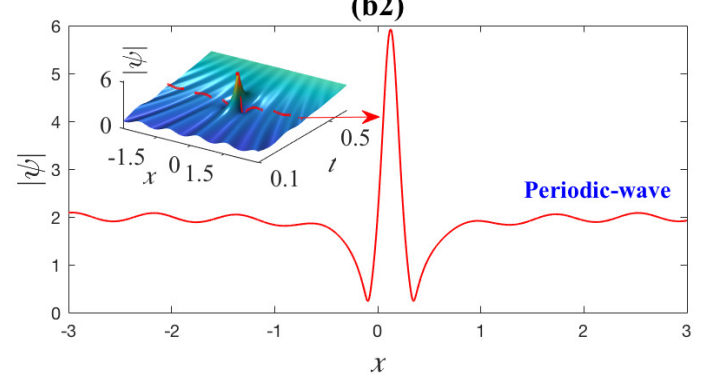

Fig. 4 (Color online) (a1) The evolution of peak at $x=\frac{1}{8 \mathrm{e}^{2 t}}$ (deep red line) and depression at $x=$ $\frac{\sqrt{3} \sqrt{256 \mathrm{e}^{12 t}-768 \mathrm{e}^{8 t}+577 \mathrm{e}^{4 t}}}{8 \mathrm{e}^{4 t}}$ (blue line), and (b1) The profile map for $t=\frac{1}{4} \ln \frac{3}{2}$ of first-order RW in Fig. 1(a1); (a2) The evolution of peak at $x=0.13$ (deep red line) and depression at $x=0.34$ (blue line); (b2) The profile map for $t=0.35$ of first-order RW in Fig. 3(a1).

(V) When $m=2, n=1$ (i.e. $\Xi_{1}=\left\{\xi_{1}\right\}$ and $\Xi_{2}=\left\{\xi_{2}\right\}$ ), $m_{1}=0$, set $\alpha=0, \beta=-1, u=2, A_{c}=1$, $\omega_{c}=0, \xi_{1}=M, \xi_{2}=\frac{5 \mathrm{i}}{2}, C_{1}=-D_{1}=\frac{1}{z}, C_{2}=D_{2}=1, \mu_{0}=\nu_{0}=0$, the mixed interaction of nonautonomous RW (red arrow) and one-breather on a growing plane-wave background can be obtained, and its corresponding structure is shown in Fig. 5 (a1). The one-breather of such a kind shows a transition of nonlinear phenomena from bright soliton structure to periodic-wave structure, such as Ref. [44]. Therefore, Fig. 5 (a1) exhibits mixed interaction of nonautonomous RW and bright soliton before $t=0$, and after that, the one-breather with an increase in the peak value will appear. Changing the $\mu_{0}=18$, the nonautonomous RW becomes a common RW with a peak and two depressions. Hence, the mixed interaction structures of one-breather and first-order RW on a growing plane-wave background can be obtained, and its corresponding structure is exhibited in Fig. 5 (a2). The white circle in Fig. 5 (a2) indicates where RW appears and interacts with the breather. When the $m_{1}=0$ is changed to $m_{1}=1$, the mixed interaction of one-breather and second-order RW on a growing plane-wave background can be obtained, and its corresponding structure is exhibited in Fig. 5 (a3). The white circle in Fig. 5 (a3) 
(a1)

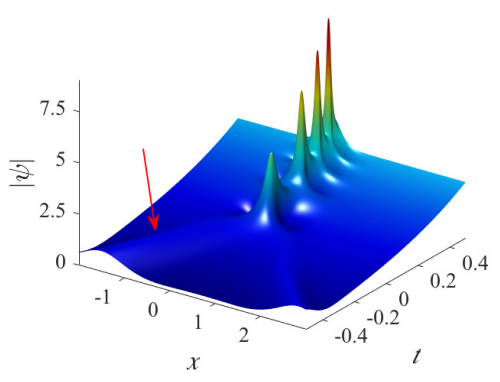

(a4)

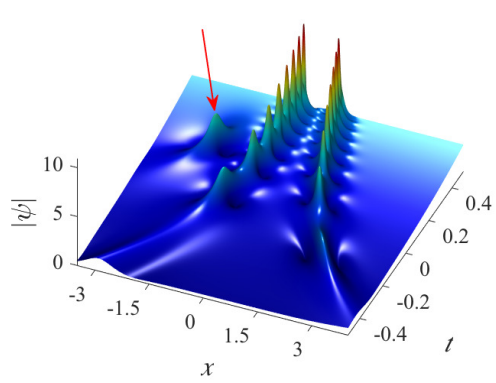

(a2)

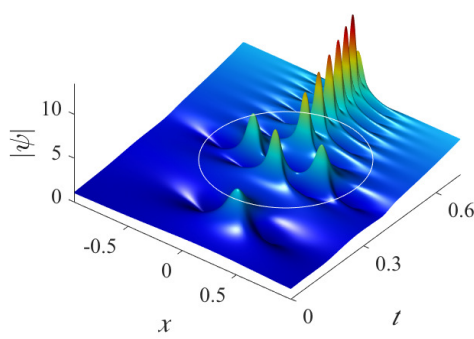

(a5)

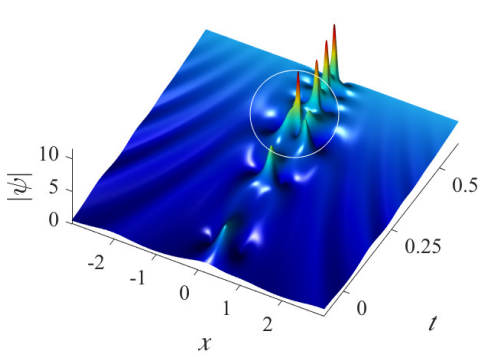

(a3)

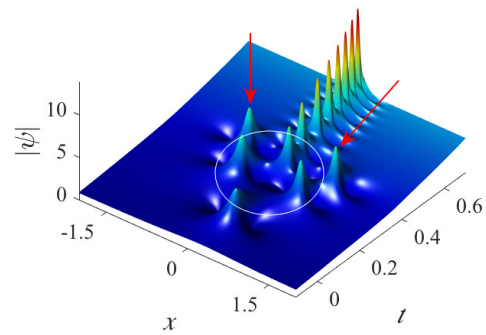

(a6)

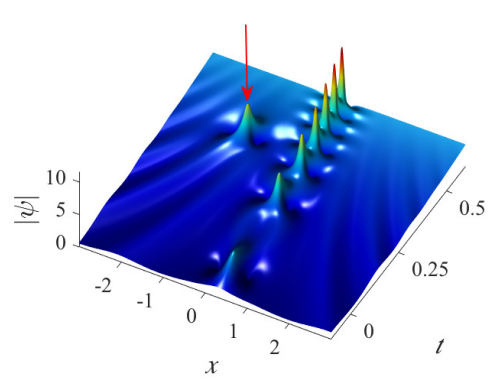

Fig. 5 Mixed interaction structures with same parameters $\alpha=0, \beta=-1, u=2, A_{c}=1, \omega_{c}=0$. (a1)-(a3) Mixed interaction of RW and nonautonomous one-breather on a growing plane-wave background with the same parameters $C_{1}=-D_{1}=\frac{C_{2}}{z}=\frac{D_{2}}{z}=\frac{1}{z}, \xi_{1}=-\frac{\omega_{c}}{2}+\mathrm{i} A_{c} u, \xi_{2}=\frac{5 \mathrm{i}}{2}$ and different parameters (a1) $\mu_{0}=\nu_{0}=0 ;(\mathrm{a} 2) \mu_{0}=18$, $\nu_{0}=0 ;$ (a3) $\mu_{0} \stackrel{z}{=} 15, \stackrel{z}{\nu_{0}}=\mu_{1}=\nu_{1}=0 ;$ (a4) Mixed interaction of RW and nonautonomous two-breather on a growing plane-wave background with the parameters $C_{1}=-D_{1}=\frac{C_{2}}{z}=\frac{D_{2}}{z}=\frac{1}{z}, \xi_{1}=-\frac{\omega_{c}}{2}+\mathrm{i} A_{c} u, \xi_{2}=-\frac{\omega_{c}}{2}+\mathrm{i} A_{c} u+\mathrm{i}$, $\mu_{0}=\nu_{0}=10$ for $\xi_{1}, \mu_{0}=\nu_{0}=\nu_{1}=0$ and $\mu_{1}=100$ for $\xi_{2}^{z}$. (a5)(a6) Mixed interaction of RW and nonautonomous one-breather on a growing periodic-wave background with the parameters $C_{1}=-D_{1}=\frac{C_{2}}{z}=\frac{D_{2}}{z}=\frac{C_{3}}{z}=-\frac{D_{3}}{z}=\frac{1}{z}$, $\xi_{1}=-\frac{\omega_{c}}{2}+\mathrm{i} A_{c} u, \xi_{2}=\frac{\mathrm{i}}{3}, \xi_{3}=\frac{5 \mathrm{i}}{2}$ and different parameters (a5) $\mu_{0}=20, \nu_{0}=0 ;$ (a6) $\mu_{0}=20, \nu_{0}=10$.

indicates where one of RWs appears and interacts with the breather and two red arrows in Fig. 5 (a3) point to where other RWs appear.

(VI) When $m=2, n=2$ (i.e. $\Xi_{1}=\left\{\xi_{1}, \xi_{2}\right\}$ and $\left.\Xi_{2}=\varnothing\right), m_{1}=0, m_{2}=1$, set $\alpha=0, \beta=-1, u=2$, $A_{c}=1, \omega_{c}=0, \xi_{1}=M, \xi_{2}=M+\mathrm{i}, C_{1}=-D_{1}=\frac{1}{z}, C_{2}=-D_{2}=1, \mu_{0}=\nu_{0}=10$ for $\xi_{1}, \mu_{0}=\nu_{0}=\nu_{1}=0$ and $\mu_{1}=100$ for $\xi_{2}$, the mixed interaction of first-order RW and two-breather structure on a growing plane-wave background can be exhibited in Fig. 5 (a4). The red arrow in Fig. 5 (a4) points to where the RW appears. It shows that two soliton-shaped waves appear initially on either side of the field, followed by two breathers. They seem to attract, and the distance between the two breathers becomes smaller, allowing RW to appear anywhere in the field via choosing appropriate parameters.

(VII) When $m=3, n=1$ (i.e. $\Xi_{1}=\left\{\xi_{1}\right\}$ and $\left.\Xi_{2}=\left\{\xi_{2}, \xi_{3}\right\}\right), m_{1}=0$, set $\alpha=0, \beta=-1, u=2, A_{c}=1$, $\omega_{c}=0, \xi_{1}=M, \xi_{2}=\frac{\mathrm{i}}{3}, \xi_{3}=\frac{5 \mathrm{i}}{2}, C_{1}=-D_{1}=\frac{1}{z}, C_{2}=D_{2}=1, C_{3}=-D_{3}=1, \mu_{0}=20, \nu_{0}=0$, the strong mixed interaction of first-order RW and one-breathers structure on a growing periodic-wave background can be exhibited in Fig. 5 (a5). Change the parameters $\nu_{0}=10$, the weak mixed interaction structure of one-breather and first-order RW on a growing periodic-wave background can be exhibited in Fig. 5 (a6). The white circle in Fig. 5 (a5) indicates where one of RWs appears and interacts with the breather and the red arrow in Fig. 5 (a6) points to where the RW appears.

In Cases (V)-(VII), one or more RWs exist in the field, and they have an interaction with the breather at any place via choosing different parameters. Forget about the RW, the dynamics of one-breather are similar to that of (IV), in that the breather propagates at an increased peak value and a decreased width. Therefore, we may use one or more RWs to act on the breather, causing it to change its amplitude or width at different times. Thus, the wave function $\psi$ can describe the dynamics of abundant localized waves and mixed localized waves interaction of bright breathers and RW in BEC with parabolic external potential.

Since the model (2) is integrable, we can obtain the infinite-order and complex nonlinear wave structures. However, for higher-order and more complex solutions, it is difficult to realize in the experiment, 
(a1)

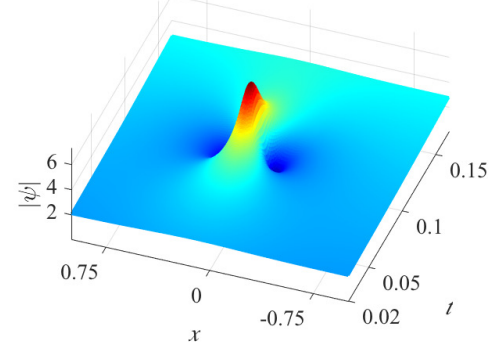

(a2)

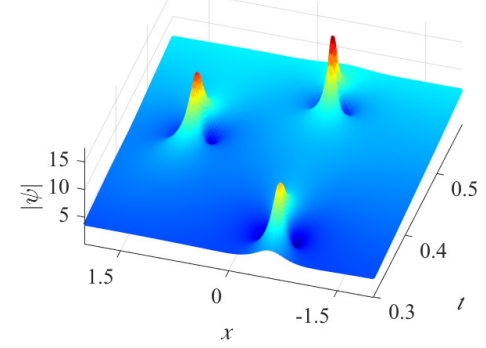

(b1)

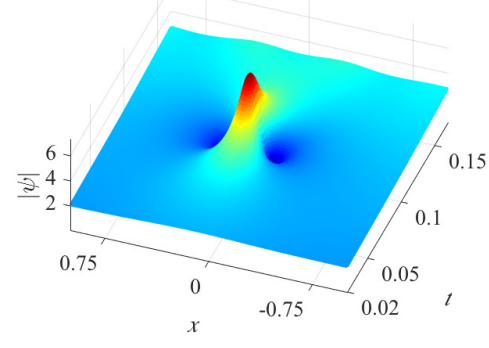

(b2)

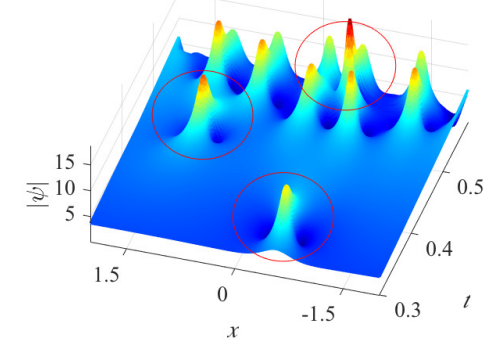

(c1)

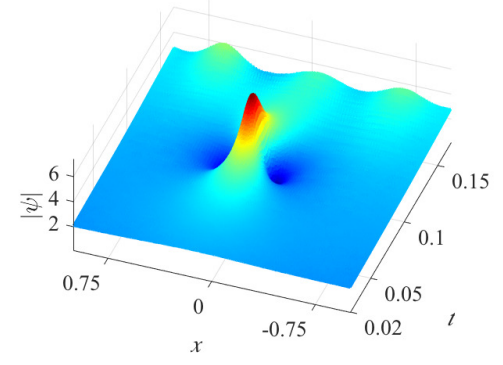

(c2)

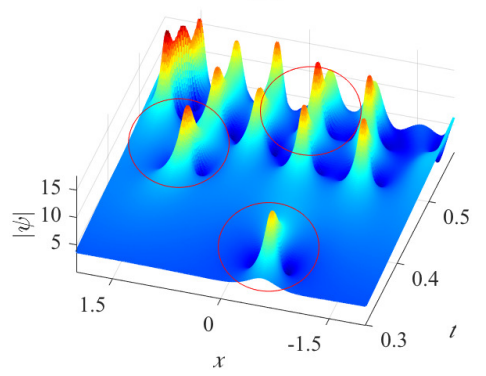

Fig. 6 Numerical simulation for RW on a growing plane-wave background with same parameters in Fig. 1(a1) (for the first row) and Fig. 1(a3) (for the second row). (a1)(a2) Exact solutions; (b1)(b2) The numerical evolution by using exact solutions at different time as an initial conditions; (c1)(c2) The numerical evolution by using exact solutions at different time with $3 \%$ perturbation as an initial conditions.

which leads to little significance of its research. Therefore, we omit the more complex nonlinear wave structure here. DT can provide a good tool to obtain all kinds of complex localized wave solutions. In addition, a significant feature of the integrable equation is that it possesses an infinite number of conservation laws [51]. Hence, the Eq. (2) also has the following conservation laws

$$
\left(\psi^{*} u_{n} \mathrm{e}^{\frac{1}{2} \beta x^{2}}\right)_{t}=\left[\left(\mathrm{i} \psi^{*} u_{n+1} \mathrm{e}^{-2 \beta t}+\frac{\alpha}{2 \beta} \psi^{*} u_{n}-\mathrm{i} u_{n} \psi_{x}^{*}-x \beta u_{n} \psi^{*}\right) \mathrm{e}^{\frac{\mathrm{i}}{2} \beta x^{2}}\right]_{x}, \quad n=1,2,3, \cdots,
$$

with

$$
\begin{aligned}
& u_{1}=u \psi \mathrm{e}^{-\frac{\beta\left(\mathrm{i} x^{2}-4 t\right)}{2}}, \quad u_{2}=\left(u_{1, x}+\frac{1}{2 \beta} \mathrm{i} \alpha u_{1}\right) \mathrm{e}^{2 \beta t}, \quad \cdots, \\
& u_{n}=\left(u \psi^{*} \mathrm{e}^{\frac{\mathrm{i} \beta \mathrm{x}^{2}}{2}} \sum_{j=1}^{n-2} u_{j} u_{n-j-1}+u_{n-1, x}+\frac{1}{2 \beta} \mathrm{i} \alpha u_{n-1}\right) \mathrm{e}^{2 \beta t},
\end{aligned}
$$

where $\psi^{*} u_{n} \mathrm{e}^{\frac{1}{2} \beta x^{2}}$ and $\left(\mathrm{i} \psi^{*} u_{n+1} \mathrm{e}^{-2 \beta t}+\frac{\alpha}{2 \beta} \psi^{*} u_{n}-\mathrm{i} u_{n} \psi_{x}^{*}-x \beta u_{n} \psi^{*}\right) \mathrm{e}^{\frac{\mathrm{i}}{2} \beta x^{2}}$ represent the density and flux, respectively and it gives infinite local conservation laws for Eq. (2). The infinite conserved quantities for the Eq. (2) is then $I_{n}=\int_{-\infty}^{\infty} \psi^{*} u_{n} \mathrm{e}^{\frac{1}{2} \beta x^{2}} \mathrm{~d} x, n=1,2,3, \cdots$. In this, the first three quantities are conservations of mass, momentum, and energy [51], they are

$$
\begin{aligned}
I_{1} & =\int_{-\infty}^{\infty} u|\psi|^{2} \mathrm{e}^{\chi} \mathrm{d} x \\
I_{2} & =\int_{-\infty}^{\infty}\left[u \psi_{x} \psi^{*}-\mathrm{i} u\left(\beta x-\frac{\alpha}{2 \beta}\right)|\psi|^{2}\right] \mathrm{e}^{\chi} \mathrm{d} x \\
I_{3} & =\int_{-\infty}^{\infty}\left[u \psi_{x x} \psi^{*}-2 \mathrm{i} u\left(\beta x-\frac{\alpha}{2 \beta}\right) \psi_{x} \psi^{*}-u|\psi|^{2}\left(-|\psi|^{2} u^{2}+\beta^{2} x^{2}+\mathrm{i} \beta-\alpha x+\frac{\alpha^{2}}{4 \beta^{2}}\right)\right] \mathrm{e}^{\chi} \mathrm{d} x
\end{aligned}
$$

with $\chi=\frac{\beta}{2}\left[(1-\mathrm{i}) x^{2}+4 t\right]$.

Numerical simulation can also confirm above analytical results. Choosing an appropriate initial condition (e.g. the exact solution (13) at $t=0.02$ ) makes the wave propagate by finite difference method. For comparison purposes, we plot the exact solution after $t=0.02$ in Fig. 6 (a1). The numerical simulation 
(a1)

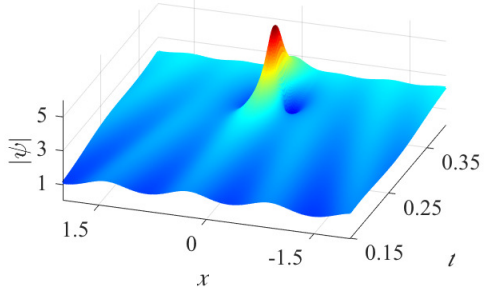

(a2)

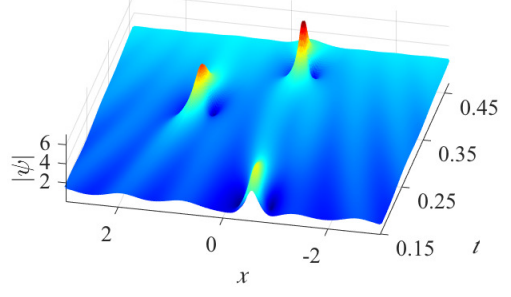

(a3)

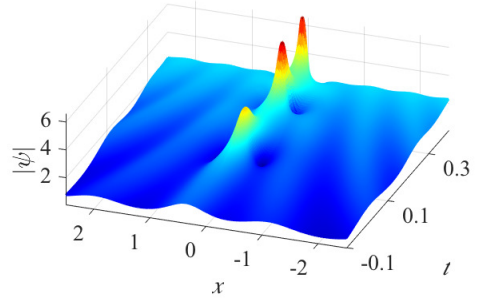

(b1)

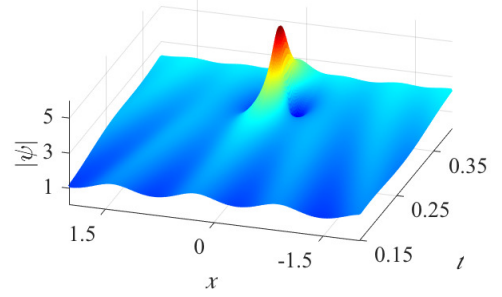

(b2)

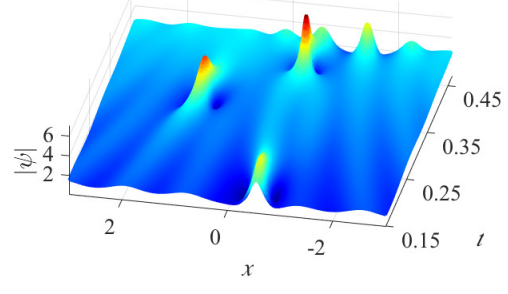

(b3)

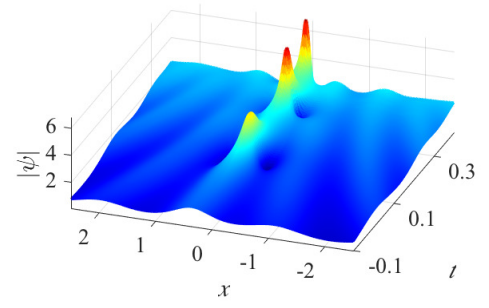

(c1)

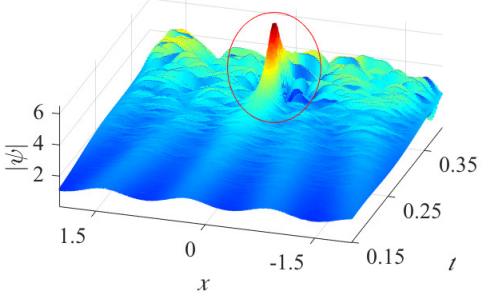

(c2)

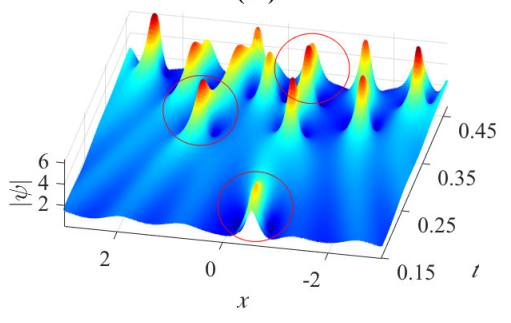

(c3)

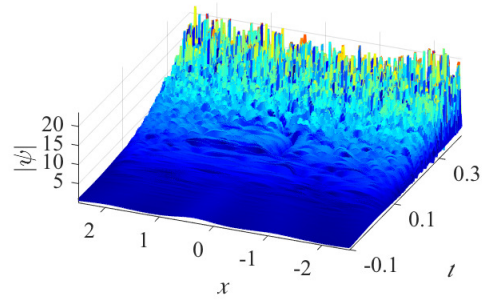

Fig. 7 Numerical simulation for RW and nonautonomous one-breather on a growing periodic-wave background with same parameters in Fig. 3(a1) (for the first row), Fig. 3(a2) (for the second row) and Fig. 3(a3) (for the third row). (a1)-(a3) Exact solutions; (b1)-(b3) The numerical evolution by using exact solutions at different time as an initial conditions; (c1)-(c3) The numerical evolution by using exact solutions at different time with $3 \%$ perturbation as an initial conditions.

results are shown in Fig. 6 (b1) completely restore the structure of the first-order RW (see Fig. 6 (a1) and Fig. 6 (b1)), hence, the numerical result proves that the numerical method and analytical solution are correct. In order to explore the structure stability of first-order RW when it is disturbed, the initial condition will be added a small-amplitude of 0.03 . The perturbed solution is used as the initial condition to make wave propagate, the numerical simulation result still shows a complete structure of the first-order RW. The background shows obvious oscillatory behavior only after the RW disappears (see Fig. 6 (c1)). Therefore, we can assume that the first-order RW is stable enough to resist small-amplitude disturbances.

Figure 6 (a2) and (b2) displays the structure of exact second-order RW solution with the same structure in Fig. 1 (a3) and numerical simulation results by choosing the exact solution at $t=0.3$ as the initial condition. The numerical simulation exhibits an arbitrary profile of a single-RW can evolve a second-order RW composed of three independent RWs. The red circles represent RW corresponding original second-order RW structure as Fig. 6 (a2). Unfortunately, near the end of the numerical evolution, a large number of oscillations occur, which we believe to be caused by an increasing plane-wave background height. Figure 6 (c2) shows the numerical simulation result by using the initial condition as Fig 6 (b2), with a small-amplitude perturbation of 0.03 added. Three RWs may occur with similar results in Fig 6 (b2). The numerical simulation results of this part show that RW can resist some small disturbances, however, plane-wave with increasing amplitude can not resist disturbances. The above theoretical research and numerical experiments can provide a good tool for studying the RW of BEC in real experiments.

Moreover, we also plot numerical simulation of first-order on a growing periodic-wave background in Fig. 7. Figure 7 (a1), (a2) and (a3) exposes the structure of exact solution and Fig. 7 (b1), (b2) and (b3) shows the numerical structure by using the corresponding exact solution at $t=0.15, t=0.15$ and $t=-0.1$ as the initial condition, respectively. Add a small-amplitude perturbation of 0.03 to their initial condition as a new initial condition, their numerical evaluations are exposed in Fig. 7 (c1), (c2) and (c3). These RW structures can still evolve in significant oscillations (see red circles), whereas breathers cannot. These exhibit the same result with Fig. 1. Small-amplitude perturbations are magnified exponentially due to the increasing background height over a period of time so that apparently large oscillations occur 
(a1)

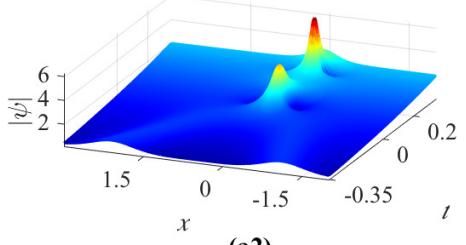

(a2)

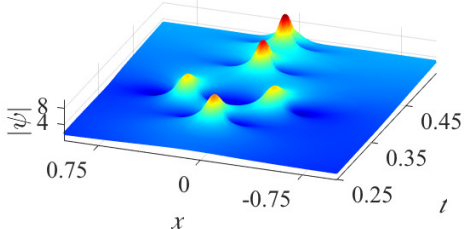

(a3)

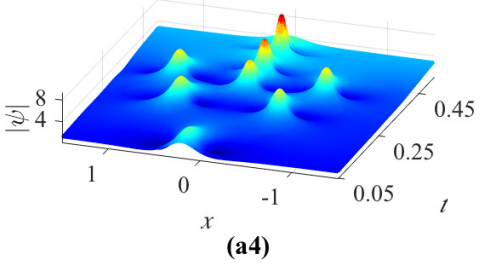

(a4)

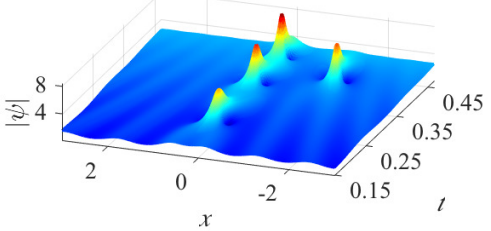

(b1)

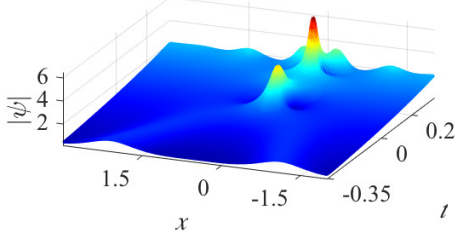

(b2)

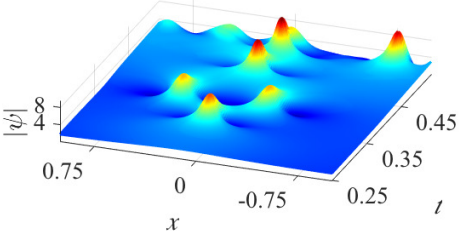

(b3)
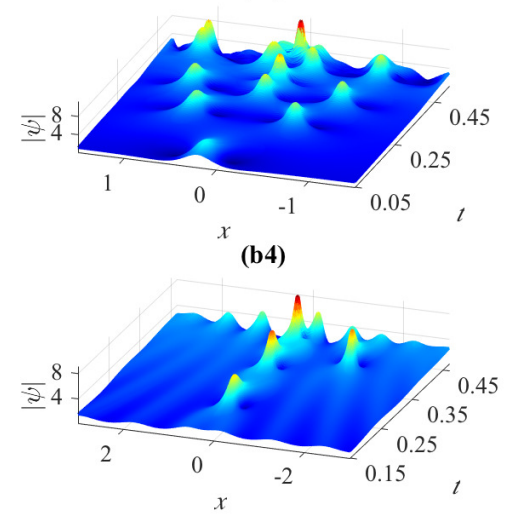

(c1)

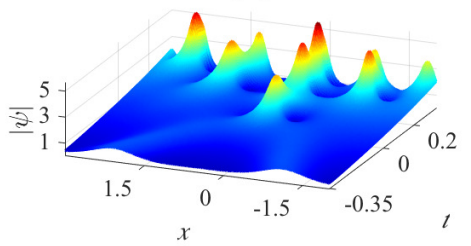

(c2)

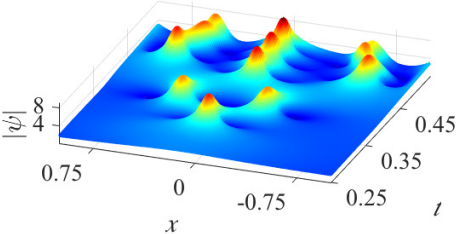

(c3)

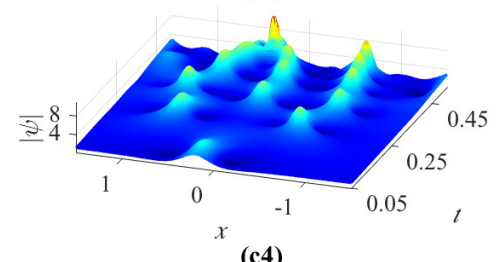

(c4)

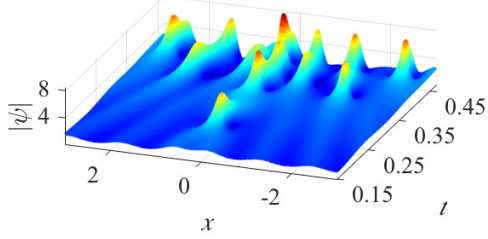

Fig. 8 Numerical simulation for mixed interaction solutions on a growing plane-wave or periodic-wave background with same parameters in Fig. 5(a1) (for the first row), Fig. 5(a2) (for the second row), Fig. 5(a3) (for the third row) and Fig. 5(a6) (for the fourth row) . (a1)-(a4) Exact solutions; (b1)-(b4) The numerical evolution by using exact solutions at different time as an initial conditions; (c1)-(c4) The numerical evolution by using exact solutions at different time with $3 \%$ perturbation as an initial conditions.

over a period of time. This oscillation of first- and second-order RW subsequently develops into a "sea" of different wave, as seen in Figs. 6(b2)(c2), 7(c1), 7(b2)(c2), among these waves, the structures excitations in the original wave function can be found (see red circles).

The numerical simulations of other solutions are shown in Fig. 8. Figure 8 (a1), (a2), (a3) and (a4) exposes the structure of exact solution and Fig. 8 (b1), (b2), (b3) and (b4) displays the numerical structure by using the corresponding exact solution at $t=-0.35, t=0.25, t=0.05$ and 0.15 as the initial condition, respectively. These numerical simulation results show that the exact solutions can arise via numerical evaluation under an appropriate initial condition. At the end of the numerical evaluation, there will be significant oscillations. Figure 8 (c1), (c2), (c3) and (c4) shows the numerical evaluation by choosing their initial condition with a small-amplitude perturbation of 0.03 , the results display these solutions may resist small perturbations for a short period of time. Due to increasing plane-wave amplitude, the solution will have enormous oscillation after a short time. The above numerical experiments show that the RW structure can be successfully evolved, and it has a certain stability, that is, it can resist the disturbance of small-amplitude. If the evolution time is longer, these oscillations will develop into a "sea" of different wave, and the original wave function structure can still be found like Figs. 6-7. This provides a well theoretical basis for the study of the RW phenomenon in BEC. Moreover, it also provides an effective theoretical method for the study of nonlinear waves in one-dimensional GPEs.

\section{Conclusions}

In conclusion, we give a set of dynamics of analytical solutions of the GPE in some different potentials with the time-varying nonlinear coefficient. These results describe macroscopic wave functions in BEC with complex nonlinear structures of higher-order RW, breather and mixed interaction solution on a variational background height in an attractive gain (or loss) term, linear potential and parabolic potential. 
Numerical simulation exhibits RW and mixed interaction solutions can resist the disturbance of smallamplitude, however, the breather can't. These may furnish an effective experimental reference for studying the validity range of the one-dimensional GPE. More importantly, the amplitude of localized waves is greatly affected by the background height. We can produce RW at any location in the field based on the parameters associated with the regulation, which also provides a good way to obtain RW with different energies and amplitudes. This makes it possible to control the nonlinear waves with different energy in the experiment in the future.

Acknowledgments This work has been supported by the National Natural Science Foundation of China (11875008,12075034); Beijing Youth Top-notch Talent Support Program (2017000026833ZK08); Fund of State Key Laboratory of Information Photonics and Optical Communications (Beijing University of Posts and Telecommunications (IPOC2019ZZ01)).

\section{Declarations}

Conflict of interest The authors declare that there is no conflict of interests regarding the publication of this paper.

Ethical approval The authors declare that they have adhered to the ethical standards of research execution.

Availability of data and material The authors declare that all data generated or analysed during this study are included in this article.

\section{Appendix}

Second-order RW solution $\psi$ in Fig. 1 (a3)(b3) is expressed as $q=-\frac{49152 F}{G} \mathrm{e}^{2 t-\frac{\mathrm{i} x^{2}}{2}+2 \mathrm{ie}^{4 t}}$, where

$$
\begin{aligned}
F= & \left.\frac{19325}{24}+\frac{3127 \mathrm{i}}{16}-\frac{1}{6} x^{6}+\left(\frac{25}{4}+\frac{\mathrm{i}}{2}\right) x^{4}-\left(\frac{1863}{16}+\frac{75 \mathrm{i}}{4}\right) x^{2}\right] \mathrm{e}^{12 t}+\left[-\frac{1905653}{512}-\frac{9775 \mathrm{i}}{8}\right. \\
& \left.-\left(\frac{627}{32}+\frac{25 \mathrm{i}}{8}\right) x^{4}+\left(\frac{1925}{4}+\frac{1875 \mathrm{i}}{16}\right) x^{2}\right] \mathrm{e}^{8 t}-\frac{1}{2} x\left[-\frac{315}{4}-\frac{75 \mathrm{i}}{4}-\frac{1}{2} x^{4}+\left(\frac{25}{2}+\mathrm{i}\right) x^{2}\right] \mathrm{e}^{10 t} \\
& +\left[-\frac{3115}{32}-\frac{125 \mathrm{i}}{8}-\frac{1}{2} x^{4}+\left(\frac{25}{2}+\mathrm{i}\right) x^{2}\right] \mathrm{e}^{16 t}+\left[\frac{9297875}{1024}+\frac{1950701 \mathrm{i}}{512}-\left(\frac{385501}{512}+\frac{3925 \mathrm{i}}{16}\right) x^{2}\right] \mathrm{e}^{4 t} \\
& +\frac{25 x}{8}\left[-\frac{13}{8}-\frac{318 \mathrm{i}}{25}+\left(\frac{206}{25}+\mathrm{i}\right) x^{2}\right] \mathrm{e}^{6 t}-\frac{1}{2} x\left(-x^{2}+\frac{25}{2}+\mathrm{i}\right) \mathrm{e}^{14 t}+\left(\frac{25}{4}+\frac{\mathrm{i}}{2}-\frac{1}{2} x^{2}\right) \mathrm{e}^{20 t} \\
& -\left(\frac{365837}{1024}-\frac{625 \mathrm{i}}{128}\right) x \mathrm{e}^{2 t}-\frac{1}{6} \mathrm{e}^{24 t}-\frac{224635711}{24576}-\frac{9674075 \mathrm{i}}{2048}+\frac{1}{4} \mathrm{e}^{18 t} x, \\
G= & \left(4096 x^{6}-153600 x^{4}+2880000 x^{2}-20172800\right) \mathrm{e}^{12 t}+\left(484608 x^{4}-12057600 x^{2}+95075952\right) \mathrm{e}^{8 t} \\
& +\left(-6144 x^{5}+153600 x^{3}-976896 x\right) \mathrm{e}^{10 t}+\left(12288 x^{4}-307200 x^{2}+2407680\right) \mathrm{e}^{16 t}+\left(19226352 x^{2}\right. \\
& -238192200) \mathrm{e}^{4 t}+\left(-635904 x^{3}+240000 x\right) \mathrm{e}^{6 t}+\left(-12288 x^{3}+153600 x\right) \mathrm{e}^{14 t}+\left(12288 x^{2}\right. \\
& -153600) \mathrm{e}^{20 t}-6144 x \mathrm{e}^{18 t}+8534712 x \mathrm{e}^{2 t}+4096 \mathrm{e}^{24 t}+248134411 .
\end{aligned}
$$

\section{References}

1. Cardoso W.B., Teixeira R.M.P.: Scattering of solitons in binary Bose-Einstein condensates with spin-orbit and Rabi couplings. Nonlinear Dyn. 96, 1147-1167 (2019)

2. Gavioli A., Sacchetti A.: On a mathematical model for a damped and driven double-well Bose-Einstein condensate. Physica D 414, 132711 (2020)

3. Wang D.S., Shi Y.R., Feng W.X., Wen L.: Dynamical and energetic instabilities of $F=2$ spinor Bose-Einstein condensates in an optical lattice. Physica D 351-352, 30-41 (2017)

4. Khawaja U.A.: Integrability of a general Gross-Pitaevskii equation and exact solitonic solutions of a Bose-Einstein condensate in a periodic potential. Phys. Lett. A 373, 2710-2716 (2009) 
5. He J.S., Charalampidis E.G., Kevrekidis P.G., Frantzeskakis D.J.: Rogue waves in nonlinear Schrödinger models with variable coefficients: Application to Bose-Einstein condensates. Phys. Lett. A 378, 577-583 (2014)

6. Kim K., Hur J., Huh S., Choi S., Choi J.Y.: Emission of spin-correlated matter-wave jets from spinor Bose-Einstein condensates. Phys. Rev. Lett. 127, 043401 (2021)

7. Kevrekidis P.G., Frantzeskakis D.J., Carretero-González R., et al.: Emergent nonlinear phenomena in Bose-Einstein Condensates. Springer-Verlag, Berlin Heidelberg (2008)

8. Wang D.S., Song S.W., Xiong B., Liu W.M.: Quantized vortices in a rotating Bose-Einstein condensate with spatiotemporally modulated interaction. Phys. Rev. A 84, 053607 (2011)

9. Dalfovo F., Giorgini S., Pitaevskii L., Stringari S.: Theory of Bose-Einstein condensation in trapped gases. Rev. Mod. Phys. 71, 463 (1999)

10. Feder D.L., Svidzinsky A.A., Fetter A.L., Clark C.W.: Anomalous modes drive vortex dynamics in confined BoseEinstein condensates. Phys. Rev. Lett. 86, 564 (2001)

11. García-Ripoll J.J., Pérez-García V.M.: Vortex bending and tightly packed vortex lattices in Bose-Einstein condensates. Phys. Rev. A 64, 053611 (2001)

12. Saito H., Ueda M.: Emergence of bloch bands in a rotating bose-einstein condensate. Phys. Rev. Lett. 93, 220402 (2004)

13. Shaukat M.I., Castro E.V., Terças H.: Quantum dark solitons as qubits in Bose-Einstein condensates. Phys. Rev. A 95, $053618(2017)$

14. Meng H., Zhou Y., Li X., Ren X., Wan X., Zhou Z., Wang W., Shi Y.: Gap solitons in Bose-Einstein condensate loaded in a honeycomb optical lattice: Nonlinear dynamical stability, tunneling, and self-trapping. Physica A 577, $126087(2021)$

15. Bhat I.A., Sivaprakasam S., Malomed B.A.: Modulational instability and soliton generation in chiral Bose-Einstein condensates with zero-energy nonlinearity. Phys. Rev. E 103, 032206 (2021)

16. Fritsch A.R., Lu M., Reid G.H., Piñeiro A.M., Spielman I.B.: Creating solitons with controllable and near-zero velocity in Bose-Einstein condensates. Phys. Rev. A 101, 053629 (2020)

17. Bludov Y.V., Konotop V.V., Akhmediev N.: Matter rogue waves. Phys. Rev. A 80, 033610 (2009)

18. Qin Z., Mu G.: Matter rogue waves in an $F=1$ spinor Bose-Einstein condensate. Phys. Rev. E 86, 036601 (2012)

19. Yu F.: Matter rogue waves and management by external potentials for coupled Gross-Pitaevskii equation. Nonlinear Dyn. 80, 685-699 (2015)

20. Trombettoni A., Smerzi A.: Discrete Solitons and Breathers with Dilute Bose-Einstein Condensates. Phys. Rev. Lett. 86, 2353-2356 (2001)

21. Cardoso W.B., Avelar A.T., Bazeia D.: Modulation of breathers in cigar-shaped Bose-Einstein condensates. Phys. Lett. A 374, 2640-2645 (2010)

22. Shomroni I., Lahoud E., Levy S., Steinhauer J.: Evidence for an oscillating soliton/vortex ring by density engineering of a Bose-Einstein condensate. Nat. Phys. 5, 193-197 (2009)

23. Rosenbusch P., Bretin V., Dalibard J.: Dynamics of a singlevortex line in a Bose-Einstein condensate. Phys. Rev. Lett. 89, $200403(2002)$

24. Bretin V., Rosenbusch P., Dalibard J.: Dynamics of a single vortex line in a Bose-Einstein condensate. J. Opt. B: Quantum Semiclass. Opt. 5, S23-S28 (2003)

25. Sulem C., Sulem P.L.: The Nonlinear Schrödinger Equation: Self-focusing and Wave Collapse. Springer-Verlag, Berlin Heidelberg (1999)

26. Alotaibi M.O.D., Carr L.D.: Internal oscillations of a dark-bright soliton in a harmonic potential. J. Phys. B: At. Mol. Opt. Phys. 51, 205004 (2018)

27. Xu T., Chen Y.: Darboux transformation of the coupled nonisospectral Gross-Pitaevskii system and its multicomponent generalization. Commun. Nonlinear Sci. Numer. Simulat. 57, 276-289 (2018)

28. Yan Z.: Two-dimensional vector rogue wave excitations and controlling parameters in the two-component GrossPitaevskii equations with varying potentials. Nonlinear Dyn. 79, 2515-2529 (2015)

29. Dai C.Q., Zhou G.Q., Chen R.P., Lai X.J., Zheng J.: Vector multipole and vortex solitons in two-dimensional Kerr media. Nonlinear Dyn. 88, 2629-2635 (2017) 
30. Sun W.R., Wang L.: Matter rogue waves for the three-component Gross-Pitaevskii equations in the spinor Bose-

Einstein condensates. P. Roy. Soc. A 474, 20170276 (2018)

31. Weidemüller M., Zimmermann C.: Interactions in ultracold gases: from atoms to molecules. Wiley-Vch, Weinheim (2003)

32. Agrawal G.: Nonlinear fiber optics. Fifth ed. Academic Press, New York (2013)

33. Kivshar Y.S., Agrawal G.P.: Optical solitons: From fibers to photonic crystals. Academic Press, New York (2003)

34. Malomed B.A., Mihalache D., Wise F., Torner L.: Spatiotemporal optical solitons. J. Opt. B: Quantum Semiclass. Opt. 7, R53-R72 (2005)

35. Solli D.R., Ropers C., Koonath P., Jalali B.: Optical rogue waves. Nature 450, 1054-1057 (2007)

36. Bailung H., Sharma S.K., Nakamura Y.: Observation of Peregrine solitons in a multicomponent plasma with negative ions. Phys. Rev. Lett. 107, 255005 (2011)

37. Onorato M., Residori S., Bortolozzo U., Montina A., Arecchi F.T.: Rogue waves and their generating mechanisms in different physical contexts. Phys. Rep. 528, 47-89 (2013)

38. Chabchoub A., Hoffmann N.P., Akhmediev N.: Rogue wave observation in a water wave tank. Phys. Rev. Lett. 106, $204502(2011)$

39. Wazwaz A.M., Kaur L.: Complex simplified Hirota's forms and Lie symmetry analysis for multiple real and complex soliton solutions of the modified KdV-Sine-Gordon equation. Nonlinear Dyn. 95, 2209-2215 (2019)

40. Wazwaz A.M.: Painlevé analysis for Boiti-Leon-Manna-Pempinelli equation of higher dimensions with timedependent coefficients: Multiple soliton solutions. Phys. Lett. A 384126310 (2020)

41. Wang L., Luan Z., Zhou Q., Biswas A., Alzahrani A.K., Liu W.: Bright soliton solutions of the (2+1)-dimensional generalized coupled nonlinear Schrödinger equation with the four-wave mixing term. Nonlinear Dyn. 104, 2613-2620 (2021)

42. Sun B., Wazwaz A.M.: General high-order breathers and rogue waves in the (3+1)-dimensional KP-Boussinesq equation. Commun. Nonlinear Sci. Numer. Simulat. 64, 1-13 (2018)

43. Yue Y., Huang L., Chen Y.: $N$-solitons, breathers, lumps and rogue wave solutions to a $(3+1)$-dimensional nonlinear evolution equation. Comput. Math. Appl. 75 2538-2548 (2018)

44. Su C.Q., Gao Y.T., Xue L., Wang Q.M.: Nonautonomous solitons, breathers and rogue waves for the Gross-Pitaevskii equation in the Bose-Einstein condensate. Commun. Nonlinear Sci. Numer. Simulat. 36, 457-467 (2016)

45. Kengne E., Lakhssassi A., Liu W.M.: Non-autonomous solitons in inhomogeneous nonlinear media with distributed dispersion. Nonlinear Dyn. 97, 449-469 (2019)

46. Sun W.R., Tian B., Jiang Y., Zhen H.L.: Double-Wronskian solitons and rogue waves for the inhomogeneous nonlinear Schrödinger equation in an inhomogeneous plasma. Ann. Phys. 343, 215-227 (2014)

47. Li L., Li Z., Li S., Zhou G.: Modulation instability and solitons on a cw background in inhomogeneous optical fiber media. Opt. Commun. 234, 169-176 (2004)

48. Tao Y.S., He J.S., Porsezian K.: Deformed soliton, breather, and rogue wave solutions of an inhomogeneous nonlinear Schrödinger equation. Chin. Phys. B 22, 074210 (2013)

49. Wen X.Y., Yang Y., Yan Z.: Generalized perturbation $(n, M)$-fold Darboux transformations and multi-rogue-wave structures for the modified self-steepening nonlinear Schrödinger equation. Phys. Rev. E 92, 012917 (2015)

50. Wang H.T., Wen X.Y.: Modulational instability, interactions of two-component localized waves and dynamics in a semi-discrete nonlinear integrable system on a reduced two-chain lattice. Eur. Phys. J. Plus 136, 461 (2021)

51. Yang J.: Nonlinear waves in integrable and nonintegrable systems. SIAM, Philadelphia (2010) 